Gregory M. Shreve*

\title{
Levels of Explanation and Translation Expertise
}

\begin{abstract}
The observable activity of translation, the series of text comprehension and text production bursts we identify as translation, is the result of the activation of complex underlying cognitive systems. In the conduct of research it is often useful to approach such complex systems using a 'levels of explanation' framework. This paper considers David Marr's (1982) three levels of explanation as they might apply to understanding translation and translation expertise more robustly. In cognitive translation studies to date, we have not really extended our understanding of expertise much past the second (algorithmic/representational) level in Marr's scheme; we have failed to grapple as effectively as we might with the problem of how the second generation computationalist expertise constructs we adopted almost twenty years ago could be integrated with, for instance, connectionist neural network models of the mind, creating a third generation of expertise models. This paper offers some frameworks laying out how that end might be achieved using, for instance, symbolic connectionism and implementational connectivism. Further, it argues that given the overtly symbolic nature of translation language processing, cognitively-oriented translation scholars are uniquely suited to benefit from approaches that bridge the divide between symbol processing models and connectionist ones.
\end{abstract}

\section{Keywords}

translation expertise; connectionism; computationalism; complex systems; levels of explanation

\section{Levels of organization and explanation}

The cognitive activity we refer to as translation could be viewed as arising from a hierarchically organized "complex system" of interacting components in the sense defined by McClamrock (1991: 185): "The standard model of the multiple levels of a complex system is a rough hierarchy, with the components of each ascending level being some kind of composite made up of the entities present at the next level down."

One of the features of such systems is that active relationships between the lower-level constituents of such a system can give rise to an aggregate or collective behavior observable at the next ascending level that is not attributable to any discrete constituent. The collective behavior emerges as a property of the system as a whole. Thus, we can say complex systems have systemic properties that we can observe and document empirically, even when it is sometimes unclear how they have arisen. Often we need significant epistemological assistance in comprehending and explaining how such system behavior emerges.

As observers of complex systems, it is possible to formulate explanations of system behavior at higher (coarser-grained) and lower (finer-grained) levels, and with a potentially indeterminate number of levels in between. The behavior of a complex system, a particular organism, or even the functioning of the mind, might then be explained at various levels of explanation depending on what entities and events are chosen as the focus of observation, what the research interest is, or the level of abstraction chosen for analysis.

\footnotetext{
* Gregory M. Shreve

Kent State University

New York University

167 North Prospect Street

Kent, Ohio 44240 USA

gshreve@kent.edu
} 
Regarding the mind as a complex system, the levels are not a disguised claim for the psychological reality of systemic hierarchy; they are instead best seen as a useful analytic device for understanding, for explaining. Using discrete levels of explanation is, thus, not a claim that the mind is physiologically partitioned into neat hierarchical levels. If it is, we have no absolute proof of such a hierarchy, and modern cognitive science is currently struggling with just such fundamental questions of the structure and function of the brain and how it gives rise to thought and consciousness.

The indeterminacy and apparent seamless unity of the actual state of the mind, however, does not deter us from making assumptions about it for the purposes of explanation and empirical verification. David Marr argues that levels of explanation are levels "at which an information processing device must be understood before one can be said to have understood it completely" (Marr 1982: 24). Verdejo/Quesada (2011: 5) describe the distinction between levels as "a fundamental point that clarifies successful explanations in cognitive psychology." Thus, the use of levels is an explanatory device employed to achieve an understanding of systems that are otherwise too complex to grasp, and where the boundaries and interactions of components are so ill defined that we might otherwise have trouble encompassing them.

This paper argues that regardless of the apparent unitary seamlessness of the system under observation, levels of explanation may still be of significant relevance for research, especially in cognitive translation studies, where our research object, we consider, is uniquely suited to such an explanatory approach because of its systemic complexity. The importance of adopting a comprehensive 'levels of explanation' perspective in cognitive translation studies has also been discussed in Shreve/Diamond (2016).

What we call translation, following the argument above, is a high-level functional, goal-oriented activity that might be seen as arising from the interaction of sub-systems of constituent entities at lower levels. There might be questions we would want to ask about translation that would best be answered at a particular level of explanation, and exploring lower levels of the system might not be productive. If we want to explore the relationship of reading activities to writing activities in a translation task, we might focus on a higher level of the system for our explanation than if we had a question about how focus of attention and saliency of perceptual cues interact with working memory, where our focus might be at a lower level of the system. If we had to explain why, after trauma to the brain, a translator might lose one of her two languages, then explanation at the level of brain architecture and neuronal arrays might be more appropriate. As Wilkinson (2014: 373) remarks: "If one has explanatory concerns that operate at a certain level, addressing them at a different level is at best, sub-optimal, and at worst, completely irrelevant or opaque."

If the focus of attention is the translating mind, then we might imagine that at a very low level of organization are the neurological processes of the brain as they implement phenomena such as language processes and memory retrieval. At a higher level of organization might be a composite goal-oriented activity, as for instance the act of translating the text itself. Between these two levels, where cognitive translation studies and cognitive science usually intersect, are perhaps multiple levels of intermediate mental processes of cognition that come online as needed during the progression of the translation task. We might enumerate the intermediate levels of explanation as including more general cognitive processes belonging to the categories Harnish (2002: 5) distinguishes: "attention, memory, learning, reasoning, problem solving, and aspects of motivation theory, action theory, perception, and language processing." Harnish's list includes many, if not all, of the types of mental processes whose interdependent operation during a task might underlie and explain the higher-level activity of translation. Even this level of explanation is still relatively high, where memory processes like storage and retrieval could be considered composites of even lower level memory processes.

From this perspective we could begin to explain translation by looking at its constituent systems and answer questions about how the entire complex activity enacted during the translation task could be carried out. We would have to specify what specific processes comprise it (algo- 
rithms, functions), how they interact, and what representations they might act upon (words, translation units, texts). Of course, cognitive scientists will recognize in this approach Marr's (1982) and Pylyshyn's (1984) framework of levels of explanation to be used when studying complex cognitive systems. Marr took an explicitly information processing (computationalist) perspective, claiming that complex systems could be explained at three levels of organization, the computational, the algorithmic/representational and the implementation level. Pylyshyn (1984) identified the semantic, the syntactic, and the physical levels.

Regardless of the computationalist context of the original formulations, there is a strong case to be made that as connectionist and situated cognition explanations of cognition become more widespread in our and other disciplines, levels of explanation still have an important place, as long as we understand that they are more about the utility of understanding "proper inter-level explanatory relations" and not necessarily about whether a "structure at a high level of description is reproduced in MR [author's note: mental representation] at a lower level of description" (Verdejo/Quesada 2011: 11).

\section{Levels of translation analysis}

From a leveled approach, at the highest level in cognitive translation studies we have to look at the main task activities of translation, the sub-tasks being carried out and the goals of those tasks. Here, for instance, we might focus (simplistically, I finesse the details) on source text (ST) comprehension, transfer, and target text (TT) production as the three main high-level task sequences.

The highest level is goal-oriented, it proceeds from a task-oriented functional viewpoint: what is being done and why? The highest level of analysis is really an abstract functional conceptualization of the activity being studied. At the middle level (or at any number of intermediate levels), we focus on the functional decomposition of the task abstraction at the higher level, specifying, for instance, the underlying fundamental cognitive processes and their associated mental representations. What do we mean here by fundamental? We mean that such processes are general cognitive processes that are not, strictly speaking, translational until they are brought online and activated during a translation task.

Note that these processes are studied on-line because what we want to understand is how these constituents (themselves abstractions created for utility of explanation) behave and interact when they are engaged as elements of a larger system activated during goal-oriented processing. It is important to understand that the output of the complex systems we observe when we watch a translator translate, e.g., with keystroke logging or eye tracking, is produced only during task performance, hence the imperative for us, as translation researchers, to constantly keep in mind the pervasive influence of the functional goal-orientation of the highest level of explanation. The socalled intermediate constituents of the system are always under the influence, impacted by, the task-related systemic constraints of higher levels. Their nature and operation is always task dependent.

Middle level analysis focuses not on a more general 'what' or 'why,' but on a more specific 'how,' and there can be a wide range of hows proposed for explaining system functions. For instance, how could translation be explained by looking at cognitive processes as computational algorithms, and with specific kinds of discrete mental representations as data structures? (from a computationalist point of view, but more on this later.)

Finally, at the lowest level, we would focus on how these activated translation task-bound processes and entailed representations are realized in the neural system of a human being. Neurons and neuronal arrays are the implementation or physical level, a specification of how proposed intermediate level representations and processes could be implemented in the human brain.

Let us consider the comprehension stage of the translation process. Comprehension is a generalizing construct that could possibly subsume several sub-processes, including lexical decoding during perception and activation of lexical entries in a lexical store (lexical access). If multi- 
ple languages are represented in the store, then appropriate lexical items must be selected (lexical selection), a process mediated via a shared conceptual store, which is also activated. Then there is syntactic parsing; morphological markers and lexical sequences are analyzed to establish logically meaningful relationships between the concepts activated during serial lexical access and selection. Those concepts, stored in long-term memory, activate other concepts and build a mental representation as we proceed through the text, very much as described in Walter Kintsch's construction-integration model (1988).

From the levels of organization perspective, we can see that we have functionally decomposed the higher-level process of comprehension into its process constituents (lexical access, syntactic parsing, propositional elaboration, long term memory retrieval, and so on, none of which are identifiably translational until they are activated during a task). These processes, which we often think of in the abstract, as if they were not always activated in a broader task context, are altered by the circumstances of their activation. Thus the nature of, for instance, lexical access may differ when activated during a bilingual task as opposed to a monolingual one. We have also specified some mental representations or data structures (the contents of lexical stores or knowledge stores, proposition-like mental representations, and situation models): once again, none of which are intrinsically translational. We have moved to the algorithmic/representational level of explanation.

If we look at current research in cognitive translation studies, it is obvious that our research interest does not extend much beyond this intermediate level of description. If we think of our levels of organization from a Marrian perspective, then our understanding of translation processes is actually relatively shallow. We study broad abstract processes (comprehension, for instance) and try to delve down to understand the interaction of a set of less abstract (but still generalizing) intermediate processes and mental representations as they engage during translation (lexical selection, for instance). We generally stop there and only now are venturing to proceed much further.

It seems to me that we really do have to extend our research interest to the implementation level and use the findings of the cognitive neurosciences to understand how the physical structure and function of the brain relates to the way a very complex higher level activity like translation could be carried out (see Maria Tymoczko's 2012 call for research in this area). Encouragingly, there has been an increasing body of work grappling with these ideas in recent years, see, for example García (2013), García et al. (2016), Shreve/Diamond (2016), and Diamond/Shreve (2010).

\section{Levels of analysis: translation expertise}

As in cognitive translation studies in general, we also have not delved much beyond the highest levels of analysis in our research on translation expertise, at least partly because expertise studies itself has always taken a relatively high level analytic stance. Early expertise research (first generation) was quite simple and high level indeed, reducing expertise to facility with problem solving (and characterized as a set of heuristic search methods). So, in this view, an expert "was someone particularly skilled at heuristic search" (Holyoak 1991: 301). A second generation of expertise researchers soon moved on to show that expertise was, while still about heuristics and problem solving, also heavily dependent on the accumulation of detailed domain knowledge, and on memory organization and inferencing patterns related to and precipitated by that accumulated knowledge. They argued that experts learned to do things well with the specific knowledge they accumulate via practice, hence procedural learning became an important focus of this second generation research.

Second generation theories dominated expertise studies for years, and they are the theoretical approaches most recently imported into translation studies (see Shreve 2002 among early adopters). These early approaches to expertise are primarily computationalist at heart. Risku (1998) was the exception, advocating very early for a more connectionist perspective. For most second generation theories, many basic notions (e.g., chunking, automaticity) are explained from a production rule-system perspective, a classic computationalist paradigm. Second generation theories 
proposed many of the nine general axioms of expertise we accept today (the axioms are adapted from Feltovich et al. 2006; they are listed below with some key concepts related to each):

1. Expertise is task-specific and therefore limited to the task domain (localized task domain; restrictions are based on experiential history; lack of transfer to other task domains).

2. Knowledge and content matter are important to expertise (the more you know, the more you are able to know, e.g., there is enhanced learning capability in the domain; increased knowledge precipitates cognitive changes, this may include such changes as proceduralization, optimization of memory retrieval, and so on).

3. Expertise involves larger and more integrated cognitive units ('chunking'; task-specific encoding; long term memory structures in the domain form and are linked to task-specific perceptual patterns).

4. Expertise involves functional, abstracted representations of presented information (abstracted representation of patterns and problems; problems as exemplars of more abstract classes; elaborated mental models of current task situation; schematization).

5. Expertise involves automated basic strokes (practice can produce automatisation of lower-level operations and allow development of higher-level skills and allocation of effort to metacognition and executive control).

6. Expertise involves the selective access of relevant information (changes in focus of attention during the task; ability to perceive the relevance of salient cues; improved problem identification and recognition of salient patterns).

7. Expertise involves reflection (greater task awareness; changes in nature and scope of metacognition; increased planning activity; greater process control).

8. Expertise is adaptive (progressive adaptation; restructuring of cognitive resources; reorganization of schemas; alterations in chunking).

9. Simple experience is not sufficient for the development of expertise (deliberate practice; motivational factors).

\section{Broad generalizations}

There are exceptions to these mainstays of expertise theory, see Keith J. Holyoak (1991) for some caveats and provisos relative to these axioms. As Holyoak (1991: 303-310) argues, there are cases where the empirical data provide some useful counter-evidence. For instance, sometimes experts do not perform better than novices. Sometimes experts do not work more efficiently and efficaciously, but actually work harder (see Scardamalia and Bereiter 1983 on writing tasks as ill-defined problems); they exhibit more planning and problem solving than novices or less-skilled individuals.

While we cannot comment on all of the exceptions to these axioms of expertise here, Holyoak's point is that these maxims, while useful, present a kind of monolithic view of expertise, a set of one size fits all characteristics supposedly applicable to all task domains, including translation. However, as Holyoak reminds us, the nature of the task domain and the nature of its constraints are supremely important; ultimately the nature of the task domain exerts an undeniable influence on the nature of the expertise. Expertise is not just one thing that happens in every task domain; it is inherently shaped by the nature of the tasks from which it emerges over hours of practice. There are many ways for expertise to manifest.

For instance, some scholars have argued for a distinction between routine expertise and adaptive expertise (Hatano and Inagaki 1986). Routine experts "are able to solve familiar types of problems quickly and accurately," but are less capable with "novel problems" that occur when the task varies (Holyoak 1991: 310). Adaptive experts are those that exhibit a deeper conceptual 
understanding of the task domain, and are more likely to be able to show innovation in problem solving. Adaptive expertise develops in task domains exhibiting significant variability in the task constraints, translation being a very good example.

Classical second-generation approaches to expertise adopted into translation studies typically deal with translation expertise as if it were routine expertise. There is a temptation to assume all expert translators would develop similar production rules that trigger certain actions, that they would chunk their input or organize their memories similarly, or that they would all similarly develop strong problem-resolution methods and become more advanced in recognizing domain specific patterns. There is a general presumption that expertise is not transferable from the task domain. These second-generation presumptions, given in the list of axioms above, were imported into translation studies by a number of translation scholars, including Shreve (2002). But now it is time, perhaps, to step back and reconsider.

'One-size-fits-all' may not be the case. If translation expertise is, in fact, highly adaptive as a result of its acquisition in variable and unpredictable environments, then it is something more than routine skill acquisition, it requires a deeper understanding of the nature and goals of translation to deal with variability in the task domain, variability of content, of text-type and mode of expression, of skopos and brief, and of working environment.

Empirical observations of extreme variability in the nature of expert performance strain the capacity of second-generation computationalist models of expertise to account for its highly adaptive character. Holyoak (1991) suggests that a third generation of expertise studies might help resolve the quandary expertise studies finds itself in: a much too mechanistic and simplistic conception of expertise that struggles to account for empirical evidence that expertise can be highly variable in its expression, especially in extremely variable task environments.

\section{Symbolic connectionism}

Holyoak (1991) suggests something called symbolic connectionism as a solution that (1) enhances the explanatory power of the expertise paradigm to allow for more highly variable manifestations of expertise and, perhaps more importantly, (2) provides an entrée to lower, more implementational levels of a model of expertise.

Given the extended introduction given earlier in this paper about the importance of levels of explanation, and the claim that we had not grappled sufficiently with the extension of translation studies and translation expertise into the lower implementation levels, symbolic connectionism can provide one useful avenue for us to rectify that situation.

For instance, the notion of a chunk (i.e., the knowledge structure that an expert creates and can retrieve in memory during task performance) can be given an implementation level interpretation via symbolic connectionism. We bridge from a more abstract notion at the middle levels of process understanding to a less abstract, more implementational, one at a lower level. One could conceive of a chunk, for instance, as a "tightly connected excitatory subnet of mutually supportive units" (Holyoak 1991: 315), where a unit is a neuron or cluster of neurons or "a conceptual entity related in a complex way to actual neurons" (Rumelhart et al. 1986: 329). The choice of the word 'unit' is deliberate here, these units can be neurons, neuronal arrays, or they can be structures like lexical items, concepts, or propositions that may be posited to have a complex internal neurological character.

Symbolic connectionism or similar approaches can bridge the divide between computationalist perspectives of expertise too rigid to account for the variability of empirical data and connectionist or other implementational perspectives at too a low level to readily explain research objects that encompass a multi-level complex interaction of constituent parts and processes.

Probably the best example of symbolic connectionism is that of Kintsch's (1988) construction-integration model, where the 'units' are symbolic, but the relationships between the units during text comprehension are cased in connectionist language: activation of memory concepts 
by lexical items in the text; spreading activation to close associates of the verbatim concepts; inferencing of additional propositions and the associated creation of excitatory and inhibitory links with associated weights. Kintsch is able to explain a complex phenomenon such as text comprehension, but connect it to an underlying (and perhaps empirically defensible) implementation framework. It would not be too much of a leap to construct just such a bridge in translation studies to talk about translation processing and the acquisition of translation expertise.

If we go back to the axioms listed earlier, focusing on axioms three and four, we can see that increasing expertise has always been assumed to involve the efficacious consolidation, integration and abstraction of stored knowledge about a task and its relevant constraints, the reorganization of cognitive resources. If these developmental processes have an implementational, neural expression, then we should see evidence of that in neural scans (Guida et al. 2016: 4).

Research has, in fact, provided evidence that this is just what occurs. Studies using experts and novices in different fields performing domain-specific working memory-related tasks tend to show that the brain areas activated to perform those tasks differ between novices and experts; there are changes in brain structure and function. There is even some pioneering work in interpreting studies, see for instance the work of Hervais-Adelman et al. (2017) or that of Becker et al. (2016) among others.

Guida et al. (2016: 5) have called these brain changes "functional neural reorganization," and this reorganization has the side effect of reducing overall brain activity (explaining, in part, the claims of efficiency in expertise). Functional neural reorganization refers specifically to neuroplastic changes in the human brain associated with, for instance, expertise acquisition. This reorganization can modify the way task-related brain regions interact. As explained by Bernardi et al. (2013: online):

\begin{abstract}
Functional and effective connectivity analyses revealed that ... functional changes in brain response may be accompanied also by modifications in the way task-related regions interact, usually with a strengthening of the essential couplings and a pruning of the unnecessary ones. Altogether, these observations support the so called neural efficiency hypothesis, which postulates a more efficient cortical functioning based on both a reduced utilization of resources and an improvement in information processing, thanks to a better communication between task-related brain areas, in expert/skilled as compared to ordinary individuals.
\end{abstract}

Thus, another mainstay of classical expertise studies, the restructuring of cognitive resources like schemas, for instance, can be given a neural and physiological interpretation.

We also know from the classical model of expertise that, as a result of extended (deliberate) practice, experts are assumed to develop domain-specific knowledge structures, what the literature calls chunks and schemas. Neural investigation has shown that this re-organization actually has a visible expression in the use of the medio-temporal lobe as compared to novices. For instance, "novices typically use episodic long-term memory areas (e.g., the medio-temporal lobe) for performing long-term memory tasks, experts are able to (re)use these areas also for performing working memory tasks" (Guida et al. 2016: 5). This occurs because the more working memory and long term memory interact, the more they influence one another, enabling functional neural-cerebral reorganization. In experts, in the task domain, working memory and long term memory establish what Anderson (2014: 296) called an additional "functional coalition" between parts of the brain as compared to novices. Here we can see a neural expression of deliberate practice, can we not? Deliberate practice has precipitated a change in the way the brain works, adapting it, so to speak, to the needs of the task (Bilalic 2016).

So, reiterating the main point, we have reached the point (because of developments in both models and in methods) where useful mid-level explanations of expertise (chunking, restructuring, efficiency gains) can be given an implementational, and in this case, neurological expression. 


\title{
6. Failure to launch
}

Over the last fifteen years, studies of the neural implementation of expertise have proceeded very quickly. Guida et al. (2016: 4) call the progress "prolific." Yet neither in translation studies as a whole, nor in translation expertise studies specifically, have we consistently delved down to look at lower implementation levels, whether with the finer-grained constructs of neural networks or with studies employing neural imaging (that would answer the question, at least, of what occurs physically in the brain during translation). We are only now beginning this research in earnest.

My point in this article is to make the claim that in translation studies of expertise we have, for the most part, adopted the second-generation findings and constructs of expertise research and been content with them, content to remain at the middle level of analysis. We talk about problem solving, pattern recognition, schematization, chunking, automaticity; we look at changes in memory organization, saliency of cues, and locus of attention. These are distinctly intermediate levels of analysis, and unsurprisingly, computationalist in heritage.

We need to move forward, but how? One way forward is to introduce approaches that (at least for now) bridge the gap between higher-level paradigms of translation and translation expertise to more implementational (for instance, neural network-based) perspectives. We have seen this bridging in Holyoak's work, and his championing of symbolic connectionism, but it is also seen in the work of the implementational connectivists who also try to accommodate the computationalist and connectionist paradigms (Stanford Encyclopedia of Philosophy 2015):

\begin{abstract}
They hold that the brain's net implements a symbolic processor. True, the mind is a neural net; but it is also a symbolic processor at a higher and more abstract level of description. So the role for connectionist research according to the implementationalist is to discover how the machinery needed for symbolic processing can be forged from neural network materials, so that classical processing can be reduced to the neural network account.
\end{abstract}

\section{Accommodating symbol processing, and a caveat}

Now, I want to make a caveat here. I am avoiding the extreme argument between classical computationalist paradigms and radical connectionists, the latter who would eliminate symbolic processing (and any higher levels of analysis?) from cognitive science. I am arguing that, given the state of our models of translation and translation expertise, bridging models such as symbolic connectionism and implementational connectivism serve an important explanatory function. They serve as important ways for us, with our necessarily high level interest as teachers and practitioners in the translation activity, to correlate and integrate what we can observe (and have observed, for decades) with what we are recently discovering about the structure and function of the brain.

We are, perhaps, if not bound, then at least extremely well-suited to use these bridging approaches, or other so-called "hybrid approaches" (Stanford Encyclopedia of Philosophy 2015), given our commitment to indispensable symbolic notions like word, text, sentence, and meaning, all of which are higher-level aggregates of what is undoubtedly a much messier and more complex neuro-cognitive reality.

In translation studies we tend (and have historically tended) to approach language and translation explicitly as a symbol processing problem, and maybe we must do so, given the complex character of the research problem we are attempting to resolve, and its quintessentially symbolic nature. Certainly we have a long disciplinary history of talking about translation as symbol processing that we cannot easily discard. It is by this time part of the core conceptual apparatus of our discipline, and perhaps indispensable as a form of explanation and analysis. This article argues for a continuation of the trend in our discipline to adopt a sort of Langackerian understanding of constructs like 'word' and see them also as symbolic points of access ('access nodes') to underlying knowledge networks, and, ultimately, neuronal structures (Langacker 1987: 161-164).

So we may be more inclined to find success in drilling down to the lower levels of the translation activity if we can find an approach to help us account for translation-oriented language sym- 
bol processing at the level of brain architecture. Implementationalists are our natural allies because they explicitly recognize that symbolic systems and connectionism are distinct levels of description, but argue that the two levels of description need one another.

For instance, pattern recognition is very well explained in connectionist models; changes in pattern recognition ability are often seen as fundamental to the acquisition of expertise. From neural network models of pattern recognition we have identified and explained important properties such as graceful degradation, noise tolerance, and the ability to generalize. These are also potentially important aspects of expertise to consider. Such properties have been remarked in the computationalist symbol processing literature, but the older paradigm lacked any robust explanation as to how these phenomena might arise from a neural substrate. So to a great extent, connectionism and neural network models, or other lower level models, can provide additional explanatory power to our existing models. More importantly, connectionist or other possible implementation level explanations might also more closely mirror natural or physical reality, since one could, ostensibly, more easily find neuro-physiological evidence for them. We need to be mindful of promoting cognitive theories or models that have no possible point of attachment to the realities of brain structure and function.

One way of achieving this goal of having models and theories with both explanatory and predictive power and some connection to the actual operation of the brain is to seek connections between our higher-level symbolic models and those detailing the minutiae of neural interaction. However, a problem for translation scientists looking for implementation level explanations is this: given the inextricable relationship of translation processes to language processes, how do we not get lost? The answer (which also coincides with our understanding of expertise) is to focus on the task and its unique character and constraints.

The translation unit, we argue, is the smallest unit of behavior that can be studied where the underlying goal-orientation of the task exerts an influence. It is the minimal task decomposition possible before we are studying something else other than translation, as for instance $\mathrm{L}_{2}$ reading or native language $\left(\mathrm{L}_{1}\right)$ production. If we are going to look at how translation arises from underlying neural mechanisms, then we have to study how those mechanisms are engaged during bona fide translation tasks and focus on translation units as the primary object of research interest.

One of the values of neural network models, as we have alluded, is that they help us understand some things better than classical models do. For instance, schemas (forms of memory organization/representation) have been an important part of expertise studies. Neural networks provide a useful explanation for how schemas arise. Rumelhart et al. (1986), for instance, argued that they were emergent states of neural networks. A schema is not a single neuron, but neurons working together can realize a schema (McClelland 2010).

When we adopt a multi-level system of explanation that breaks down complex systems into smaller units, then one can use emergence as a way to explain properties of mid-level constructs that are otherwise mysterious. Connectionists have argued that words and grammar rules are no more than emergent properties of clusters of simple processing units (neurons) whose connection weights are affected by experience due to hearing spoken language (McClelland 2010). Arguably, then, concepts are clusters of sub-networks; and propositions emerge from networks of sub-network clusters. Finally, a network of networks represents our understanding of a text, all emergent as the component units, nodes and neurons, activate and interact serially and in parallel during the online task.

We have already seen how chunking and restructuring can be interpreted as effects of neuronal systems being trained over long periods of time in a discrete task. Automaticity, a mainstay of expertise models, can also be understood as emerging from task repetition (see Cohen et al. 1990 where performance differences in a Stroop task were accounted for by differences in experiential inputs into a connectionist network).

Even expert metacognition could be given a neural network interpretation, as a state arising from the accumulation of task experience in a metacognitive memory network supporting, for in- 
stance, improved task awareness, judgments of success and failure, and uncertainty management. Certainly there has been progress in understanding the physiological and neural bases of metacognition, as in the work of Fleming/Dolan (2012) who concluded that there are potentially separable brain systems for prospective and retrospective judgments of performance. Thus, almost all of the important aspects of translation expertise from chunking through to metacognition could be attached to physiological structures and locations, and be at a deeper level of explanation.

The contribution of this paper, rather, is in the argument that our object of study, highly complex and explicitly symbolic by its very nature, profits greatly from the adoption of an explicitly leveled approach to explanation and bridging approaches that successfully connect task-bound mid-level constructs like translation processes to constructs like neural networks and from there, potentially, to physical brain-level structures.

Expertise in translation, and in general, arises as a result of the interactions of the many, many neurons that are brought online to accomplish a particular task, a task repeated over and over again, via deliberate practice, until performance is consistently superior. Translation expertise is the consequence of training a set of neural networks with the right kind of data over the requisite amount of time. But, even if that statement is true, and I believe that it is, explaining the acquisition of expertise and its expression at the level of the task fruitfully and comprehensively across disciplines might require a multi-level descriptive apparatus, like that proposed initially by Marr (1982).

\section{Conclusion}

Marr's highest level of explanation emphasizes the goal-oriented computational task. But we can also imagine the utility of levels of explanation above the computational also being accommodated by his approach. As Muñoz (2010) and Risku (2014) have successfully argued, cognitive processing in translation (and by extension translation expertise) cannot be understood completely unless we account for the embedded nature of cognition; it occurs within a physical, social and cultural environment; cognition has a property of situatedness that we cannot ignore. The translation activity we observe ethnographically (in its natural setting) is invoked and provoked by socio-cultural factors and processes, and they, acting through the translator as social actor, provoke a specific contextually bound kind of cognition.

The internal processes of a discrete act of translating and the active external social and communicative processes of the context of the act intersect at the task. Thus, the task remains an important focus of translation and expertise research. The serial bursts of text comprehension (reading) and text production (writing) we observe when someone translates, translation units, are the behavioral manifestation of an underlying cognitive dynamic prompted by a task, a situated socio-cognitive demand placed on an actor.

Even as we extend our understanding of expertise down to the neural level, we must also recognize that it is necessary to extend upwards. It is all processes within processes, and thus, potentially, levels of explanation within levels of explanation. When we seek explanations of how any task can be carried out, we can go quite possibly very much further down in levels of explanation; the neuron networks of the brain are not the lowest level of consideration. We could proceed to the cellular, the molecular, the atomic, and, perhaps, the quantum. As we move up in levels of explanation from the situated task, we could also go quite a lot further up, from the social and cultural perhaps all the way to the cosmological. But, as a matter of necessity, we circumscribe our levels of explanation, restricting our interests to those task-adjacent levels that contribute materially to the robustness of the explanation we wish to furnish. 


\section{References}

Anderson, Michael L. 2014: After Phrenology: Neural Reuse and the Interactive Brain. Cambridge, MA: MIT Press.

Becker, Maxi/Schubert, Torsten/Strobach, Tilo/Gallinat, Jurgen/Kühn, Simone 2016: Simultaneous Interpreters vs. Professional Multilingual Controls: Group Differences in Cognitive Control as well as Brain Structure and Function. In Neuroimage, 134, 250-260.

Bernardi, Giulio/Ricciardi, Emiliano/Sani, Lorenzo/Gaglianese, Anna/Papasogli, Alessandra/Ceccarelli, Riccardo/ Franzoni, Ferdinando/Galetta, Fabio/Santoro, Goebel/Pietrini, Pietro 2013: How Skill Expertise Shapes the Brain Functional Architecture: An fMRI Study of Visuo-Spatial and Motor Processing in Professional Racing-Car and Naïve Drivers. In PLOS-ONE, 8:10 [online]. https://doi.org/10.1371/journal.pone.0077764 (accessed 15 September 2017).

Bilalić, Merim Bilalić 2017: The Neuroscience of Expertise. Cambridge: Cambridge University Press.

Cohen, Jonathan D./Dunbar, Kevin/McClelland, James L. 1990: On the Control of Automatic Processes: A Parallel Distributed Processing Account of the Stroop Effect. In Psychological Review 97:3, 332-361.

Diamond, Bruce J./Shreve, Gregory M. 2010: Neural and Physiological Correlates of Translation and Interpreting in the Bilingual Brain: Recent Perspectives. In Shreve, Gregory/Angelone, Erik (eds.), Translation and Cognition, American Translators Association Scholarly Monograph Series XV. Amsterdam/Philadelphia: John Benjamins, 289-321.

Feltovich, Paul J./Prietula, Michael J./Ericsson, K. Anders 2006: Studies of Expertise from Psychological Perspectives. In Ericsson, K. Anders/Charness, Neil/eFeltovich, Paul J./Hoffman, Robert R. (eds.), Cambridge Handbook of Expertise and Expert Performance. Cambridge: Cambridge University Press, 41-67.

Fleming, Stephen M./Dolan, Raymond J. 2012: The Neural Basis of Metacognitive Ability. In Philosophical Transactions B 367:1594, 1338-1349.

García, Adolfo M. 2013: Brain Activity During Translation: A Review of the Neuroimaging Evidence as a Testing Ground for Clinically-based Hypotheses. In Journal of Neurolinguistics 26:3, 370-383.

García, Adolfo M./ Mikulan, Ezequiel/Ibáñez, Agustín 2016: A Neuroscientific Toolkit for Translation Studies. In Muñoz Martín, Ricardo (ed.), Reembedding Translation Process Research. Amsterdam/Philadelphia: John Benjamins, 21-46.

Guida, Alessandro/Campitelli, Guillermo/Gobet, Fernand 2016: Becoming an Expert: Ontogeny of Expertise as an Example of Neural Reuse. In Behavioral and Brain Sciences 39, 1-14.

Harnish, Robert M. 2002: Minds, Brains, Computers. An Historical Introduction to the Foundations of Cognitive Science. Oxford, England: Blackwell.

Hatano, Giyoo/Inagaki, Kayoko 1986: Two Courses of Expertise. In Stevenson, Harold/Azuma, Hiroshi/Hakuta, Kenji (eds.), Child Development and Education in Japan. New York: W.H. Freeman, 263-272.

Hervais-Adelman, Alexis/Moser-Mercer, Barbara/Murray, Micah M./Golestani, Narly 2017: Cortical Thickness Increases After Simultaneous Interpretation Training. In Neuropsychologia 98, doi:10.1016/j.neuropsychologia.2017.01.008.

Holyoak, Keith J. 1991: Symbolic Connectionism: Toward Third-Generation Theories of Expertise. In Ericsson, K. Anders/Smith, Jaqui (eds.), Toward a General Theory of Expertise. Cambridge: Cambridge University Press, 301-334.

Kintsch, Walter 1988: The Role of Knowledge in Discourse Comprehension: A Construction-integration Model. In Psychological Review 95:2, 163-182.

Langacker, Ronald 1987: Foundations of Cognitive Grammar: Theoretical Prerequisites. Stanford: Stanford University Press.

Marr, David 1982: Vision. San Francisco: Freeman.

McClamrock, Ron 1991: Marr's Three Levels: A Re-evaluation. In Minds and Machines 1:2, 185-196.

McClelland, James L. 2010: Emergence in Cognitive Science. In Topics in Cognitive Science 2:4, 751-770.

Muñoz Martín, Ricardo 2010: On Paradigms and Cognitive Translatology. In Shreve, Gregory/Angelone, Erik (eds.), Translation and Cognition. Amsterdam: John Benjamins, 169-187.

Pylyshyn, Zenon W. 1984: Computation and Cognition. Cambridge, Mass., MIT Press.

Rumelhart, David E./Smolensky, Paul/McClelland, James L./Hinton, Geoff E. 1986: Parallel Distributed Emergence. In McClelland, James L./Rumelhart, David E. (eds.), Parallel Distributed Processing: Explorations in the Microstructure of Cognition. Volume II. Cambridge, MA: MIT Press, 7-57.

Rumelhart, David E./McClelland, James L. 1986: Parallel Distributed Processing. Explorations in the Microstructure of Cognition. Cambridge, MA: MIT Press.

Risku, Hanna 2014: Translation Process Research as Interaction Research: From Mental to Socio-Cognitive Processes. Monti Special Issue 1, 331-353. 
Risku, Hanna 1998: Translatorische Kompetenz. Kognitive Grundlagen des Übersetzens als Expertentätigkeit. Tübingen: Stauffenburg.

Scardamalia, Marlene/ Bereiter, Carl 1983: The Development of Evaluative, Diagnostic and Remedial Capabilities in Children's Composing. In Martlew, Margaret (ed.), The Psychology of Written Language, Developmental and Educational Perspectives. New York, NY: Wiley, 67-95.

Shreve, Gregory M. 2002: Knowing Translation: Cognitive and Experiential Aspects of Translation Expertise from the Perspective of Expertise Studies. In Riccardi, Alessandra (ed.), Translation Studies: Perspectives on an Emerging Discipline. Cambridge: Cambridge University Press, 150-171.

Shreve, Gregory M./Diamond, Bruce J. 2016: Cognitive Neurosciences and Cognitive Translation Studies: About the Information Processing Paradigm. In Gambier, Yves/Doorslaer, Luc van (eds.), Border Crossings: Translation Studies and Other Disciplines, Amsterdam and Philadelphia: John Benjamins, 141-168.

Stanford Encyclopedia of Philosophy 2015: Connectionism. Stanford Encyclopedia of Philosophy [online]. https:// plato.stanford.edu/entries/connectionism (accessed 10 March 2017).

Tymoczko, Maria 2012: The Neuroscience of Translation. In Brems, Elke/Meylaerts, Reine/van Doorslaer, Luc (eds.), The Known Unknowns of Translation Studies. Amsterdam: John Benjamins, 83-102.

Verdejo, Victor M./Quesada, Daniel 2011: Levels of Explanation Vindicated. Review of Philosophy and Psychology [online]. DOI: 10.1007/s13164-010-0041-0 (accessed 17. October 2017).

Wilkinson, Sam 2014: Levels and Kinds of Explanation: Lessons from Neuropsychiatry. Frontiers in Psychology 5:1, 373. 